\title{
Shared decision-making in antihypertensive therapy: a cluster randomised controlled trial
}

\author{
Iris Tinsel ${ }^{{ }^{*}}$, Anika Buchholz ${ }^{2}$, Werner Vach³ ${ }^{3}$ Achim Siegel ${ }^{1}$, Thorsten Dürk ${ }^{1}$, Angela Buchholz ${ }^{4}$,
} Wilhelm Niebling ${ }^{1}$ and Karl-Georg Fischer ${ }^{5}$

\begin{abstract}
Background: Hypertension is one of the key factors causing cardiovascular diseases. A substantial proportion of treated hypertensive patients do not reach recommended target blood pressure values. Shared decision making (SDM) is to enhance the active role of patients. As until now there exists little information on the effects of SDM training in antihypertensive therapy, we tested the effect of an SDM training programme for general practitioners (GPs). Our hypotheses are that this SDM training (1) enhances the participation of patients and (2) leads to an enhanced decrease in blood pressure (BP) values, compared to patients receiving usual care without prior SDM training for GPS.
\end{abstract}

Methods: The study was conducted as a cluster randomised controlled trial (CRCT) with GP practices in Southwest Germany. Each GP practice included patients with treated but uncontrolled hypertension and/or with relevant comorbidity. After baseline assessment (T0) GP practices were randomly allocated into an intervention and a control arm. GPs of the intervention group took part in the SDM training. GPs of the control group treated their patients as usual. The intervention was blinded to the patients. Primary endpoints on patient level were (1) change of patients' perceived participation (SDM-Q-9) and (2) change of systolic BP (24h-mean). Secondary endpoints were changes of (1) diastolic BP (24h-mean), (2) patients' knowledge about hypertension, (3) adherence (MARS-D), and (4) cardiovascular risk score (CVR).

Results: In total 1357 patients from 36 general practices were screened for blood pressure control by ambulatory blood pressure monitoring (ABPM). Thereof 1120 patients remained in the study because of uncontrolled (but treated) hypertension and/or a relevant comorbidity. At T0 the intervention group involved 17 GP practices with 552 patients and the control group 19 GP practices with 568 patients. The effectiveness analysis could not demonstrate a significant or relevant effect of the SDM training on any of the endpoints.

Conclusion: The study hypothesis that the SDM training enhanced patients' perceived participation and lowered their BP could not be confirmed. Further research is needed to examine the impact of patient participation on the treatment of hypertension in primary care.

Trial registration: German Clinical Trials Register (DRKS): DRKS00000125

Keywords: Hypertension, Shared decision-making, Ambulatory blood pressure monitoring, Educational training, Primary care, Family medicine, Cluster randomised controlled trial

\footnotetext{
* Correspondence: iris.tinsel@uniklinik-freiburg.de

${ }^{1}$ Department of Medicine, Division of General Practice, University Medical

Centre Freiburg, Elsässerstr 2m, Freiburg 79110, Germany

Full list of author information is available at the end of the article
} 


\section{Background}

Hypertension is one of the key factors causing cardiovascular diseases (CVD) [1] which make up the most frequent cause of death in industrialised nations [2]. Though effective antihypertensive medication is available, a substantial rate of treated hypertensive patients exceed the (systolic/diastolic) thresholds of $140 / 90 \mathrm{mmHg}$ in clinical blood pressure measurement (CBPM) and are characterised as uncontrolled (but treated) hypertensive. Rates of controlled treated hypertension (below 140/90 $\mathrm{mmHg}$ ) range from $43 \%$ to 70\% in North America [3-8], 49\% in Switzerland [9] and between $25 \%$ and about $60 \%$ in Germany [10-12]. Long term consequences of uncontrolled hypertension like stroke or heart attack lead to high individual and societal burden.

There are two primary reasons to enhance patients' active participation in medical decisions: (1) Patients have the right to be fully informed on chances and risks of treatments $[13,14]$ and (2) therapies are presumed to be more successful if patients are involved in decisions [15-17]. One concept to achieve this is shared decision making (SDM). SDM interventions are recommended when there are several similarly effective treatment options. This applies to the treatment of uncomplicated arterial hypertension $[1,18,19]$. Although there is substantial research on SDM, until now there exists little information on the effects of SDM training for general practitioners (GPs) on patient participation and clinical outcomes [15-17,20-25]. This holds also for blood pressure (BP) in antihypertensive treatment.

The aim of this study was to implement an evaluated SDM training programme [26] for GPs within the context of hypertension treatment, and to answer the following research questions: Does the SDM training for GPs (1) enhance patients' perceived participation and (2) lower the BP values of patients?

\section{Methods}

Trial design and participants

The study was conducted as a cluster randomised controlled trial (cRCT). Each GP practice with its patients was considered as one cluster. As the intervention (SDM training for GPs) affects a GP's communication style which, in turn, affects all patients of a given GP, a randomisation on patient level would have led to a contamination of treatment conditions. While the intervention took place on GP level, the objectives of the intervention (e.g. enhanced participation and BP decrease) pertained to the patient level. In this study we used ambulatory blood pressure monitoring (ABPM) because of its higher validity compared to CBPM $[18,27,28]$. Before the first data assessment all participating GP practices were equipped with identical ABPM instruments (Mobil-O-Graph new generation 24h ABP-Control) $[29,30]$. Under conditions of ABPM a different definition of hypertension is required $[1,28,31]$. Therefore, in our study any patient is characterised as (uncontrolled) hypertensive whose $24 \mathrm{~h}$ mean is $\geq 130 / 80 \mathrm{mmHg}$ or whose daytime mean is $\geq 135 / 85 \mathrm{mmHg}$ or whose night mean is $\geq 120 / 70 \mathrm{mmHg}$.

\section{Inclusion and exclusion criteria}

The eligibility criteria for GPs were (1) location in Southwest Germany, (2) offering the full spectrum of a family doctor's health care services and (3) nonparticipation in another study implementing an SDM training as intervention. There were no other inclusion or exclusion criteria for GP practices. Most of the participating GPs belonged to teaching practices associated with the Division of General Practice of the University Medical Centre Freiburg (Germany).

Inclusion criteria for patients to be screened at $\mathrm{T} 0$ were (1) repeated prescription of antihypertensive medication, (2) age of at least 18 years, (3) insured by a statutory health fund with the exception of 'Bundesknappschaft and See-health insurance', and (4) understanding of the German language. Exclusion criteria were dementia, mental handicap, or short life expectancy. There were no other inclusion or exclusion criteria for patients.

\section{Data assessment}

Data were collected at four points (T0 - T3). Baseline data (T0) were assessed between June and December 2009. Patients with uncontrolled hypertension (defined as $24 \mathrm{~h}$ mean $\geq 130 / 80 \mathrm{mmHg}$ or daytime mean $\geq 135 / 85 \mathrm{mmHg}$ or night mean $\geq 120 / 70 \mathrm{mmHg}$ ) or with a relevant cardiovascular comorbidity (defined as diabetes mellitus, coronary heart disease $[\mathrm{CHD}] /$ heart attack, stroke/transient ischemic attack [TIA] or peripheral arterial occlusive disease [PAOD]) were then included in the study population.

Data of the three follow-up assessments (T1, T2 and T3) were collected at 6,12 and 18 months ( \pm 2 months). The last follow-up measurement (T3) was completed in September 2011. After completing the baseline assessment (T0), GP practices were randomised to either intervention (SDM training) or control group (no training, consultation as usual).

In each of the four data assessments clinical data and self-reported patient data were gathered.

Clinical data: Ambulatory blood pressure monitoring (ABPM) was conducted, and values of cholesterol and HbA1c were assessed by the GP practices so that the cardiovascular risk (CVR) of the patients could be calculated.

After each ABPM, the BP protocol was reviewed in a separate consultation and necessary therapy adaptations were decided.

Self-reported patient data: In each of the four data collections, questionnaires were distributed to the patients by their GP practices. These questionnaires contained a 
validated German instrument to survey patients' perceived participation in medical decisions (SDM-Q-9) [32], the translated and validated Medication Adherence Report Scale (MARS-D) [33], and an instrument to assess patients' knowledge about hypertension (eight items; developed by our research group). Furthermore, the questionnaires referred to changes in antihypertensive therapy and treatment outside the GP practice, to socio-demographic data, tobacco consumption, height and weight. Moreover some other self-reporting instruments [34-37] were included. All scores of the questionnaire instruments with Likert scales were transformed into a scale ranging from 0 (lowest level) to 100 (highest level).

\section{Intervention}

Those GPs who had been allocated to the intervention group took part in an SDM training programme [26] which had been evaluated in various studies [22,23,38,39]. It was adapted to the requirements of antihypertensive treatment in general practice and entailed six hours in total. The training included the following elements: (1) information on arterial hypertension, (2) physician-patient communication and risk communication, (3) the process steps of SDM, (4) motivational interviewing [40,41], (5) introduction of a decision table listing options to lower CVR, and (6) use of case vignettes for role plays simulating physician-patient consultations. Additionally, we recommended implementing a cardiovascular risk calculator for GPs which included elements of SDM $[24,42]$. Furthermore we delivered patient information flyers [43] to the GPs of the intervention group. The intervention took place before the $\mathrm{T} 1$ data assessment.

GPs of the control group treated their patients as usual.

\section{Outcomes}

The outcomes of the study pertained to the patient level. Primary endpoints were (1) change of patients' perceived participation from T0 to T1, T2 and T3 and (2) change of systolic BP (24h-mean) from T1 to T2 and T3 evaluated as the mean effect over all points in time. Secondary endpoints were (1) change of diastolic BP (24h-mean), (2) change of patients' adherence, (3) change of cardiovascular risk score (CVR), each from $\mathrm{T} 1$ to $\mathrm{T} 2$ and $\mathrm{T} 3$, and (4) change of patients' knowledge on hypertension from T0 to T1, T2 and T3. All endpoints were analysed using mixed models [44]. Additionally, the proportion of patients whose BP was uncontrolled at each time point were analysed descriptively.

\section{Randomisation}

To prevent an allocation bias on cluster level and a subsampling bias on patient level, randomisation took place after the baseline assessment [45]. We randomly selected GP practices (including their patients) for the intervention or control group, respectively. The randomisation was conducted by staff members of the Division of General Practice, University Medical Centre Freiburg. No stratification was used. The patients were blinded to the allocation of the intervention (single-blinded study).

\section{Sample size and power calculation}

Power and sample size considerations have been published in the study protocol [46]. In brief, we intended to include 1200 patients in the study population so that after a $20 \%$ drop out at the practice level and a $20 \%$ drop out at the patient level we could expect to be able to include 788 patients in the final analysis. This would allow detection of effect sizes of 0.3 or 0.35 with a power of $74 \%$ or $87 \%$, respectively, assuming an ICC of 0.05 $(\mathrm{VIF}=2.15)$ and using a Bonferroni correction for the two primary outcomes.

\section{Missing data}

The primary endpoints 'change of perceived participation' and 'change of systolic blood pressure' were analysed according to the intention-to-treat (ITT) principle. According to Kriston et al. [32] missing data in SDM-Q-9 were replaced if there were at most two missing items. Imputation was realised by using the mean of the valid items (on patient level). Subsequently the raw score was calculated and transformed into a scale ranging from 0 to 100. ABPM values were considered valid for a given point in time if ABPM data of at least 7 nocturnal and 14 daytime measurements had been reported. Missing ABPM data were not replaced (for details cf. study protocol [46]). The exploration of the complete dataset showed that for some endpoints, non-response was slightly higher in the control group than in the intervention group. But overall we found no systematic patterns of missing data.

\section{Statistical analysis of endpoints}

Primary and secondary endpoints are defined as change from baseline. For SDM-Q-9 and knowledge on hypertension, the respective T0 data were considered as baseline values, since the intervention may affect both outcomes directly at the first visit after intervention (T1). In contrast systolic and diastolic BP, CVR, and MARS-D cannot be affected by the intervention before the second visit after intervention. Therefore, the respective $\mathrm{T} 1$ data were considered as baseline values for these outcomes (for details cf. study protocol [46]). The intervention effect was evaluated as mean effect over all follow-ups using a mixed effects model (adjusted for the respective baseline values of outcomes according to European Medicine Agency (EMA) guideline [47]), where repeated measurements and clustering are dealt with by random effects. All patients contributing a baseline value and at 
least one follow-up measurement were included in the mixed model analysis.

Two kinds of sensitivity analyses were performed: (1) All endpoints whose baseline values had been calculated from $\mathrm{T} 1$ data in the main analysis (BP, CVR, and MARS-D) were re-analysed by using T0 data as baseline. (2) Both primary endpoints were re-analysed adjusting for several prognostic factors (for details on analyses see study protocol [46]). A descriptive analysis was done to illustrate the proportion of patients with uncontrolled (but treated) hypertension at each time point.

Statistical programming was performed with the Statistical Analysis System (SAS) Version 9.2.

\section{Ethics}

Interested GPs and their medical assistants were informed orally and via printed material about the study and data protection. GPs who wanted to take part in the study confirmed via a written consent form. Patients were informed about the study by their GPs. If patients agreed to take part in the study, both patient and GP signed the written informed consent form. The study and the informed consent form were approved by the Ethics Committee of the University Medical Centre Freiburg. The study protocol has been published elsewhere [46].

\section{Results}

\section{Study procedure}

The recruitment and study procedure is illustrated in Figure 1. At baseline assessment (T0) we received 1433 BP protocols. The data of 76 patients were excluded because they did not fulfil the inclusion criteria (not a member of a statutory health insurance, no repeated antihypertensive medication) or because of missing questionnaires. Hence data of 1357 patients remained. Then 237 Patients (17.5\%) were excluded because they fulfilled two conditions: (1) Their hypertension was characterised as controlled treated (defined as $24 \mathrm{~h}$ mean $<130 / 80 \mathrm{mmHg}$ and daytime mean $<135 / 85 \mathrm{mmHg}$ and night mean $<120 / 70 \mathrm{mmHg}[1,31]$ ) and (2) they had no relevant comorbidity (diabetes mellitus, coronary heart disease [CHD]/heart attack, stroke/transient ischaemic attack [TIA] or peripheral arterial occlusive disease [PAOD]). Hence 1120 patients with uncontrolled but treated hypertension and/or at least one relevant comorbid disorder continued in the study.

Then, the 37 GP practices were randomised to intervention and control group. Immediately before the intervention one GP of the intervention group opted out. This GP practice did not transfer any patient data. In total 17 GP practices received the intervention, 19 GP practices belonged to the control group. These GPs treated their patients as usual. After the T0 assessment was completed and before the T1 assessment started, the intervention (SDM training) took place. In total, the analysis sample included the data of 1120 patients: 552 patients of 17 GP practices in the intervention group and 568 patients of 19 GP practices in the control group. The mean cluster size in T0 amounted to 32.5 patients in the intervention group (range 5-46) and in the control group 29.9 patients (range 8-53); the development of the cluster sizes in the course of the study is shown in Figure 1.

In the intervention group 171 patients missed at least one follow-up measurement. Thereof, 88 patients took part in T0 only and were completely lost to follow up (15.9\%). Another 83 patients took part in T0 and in at least one of $\mathrm{T} 1, \mathrm{~T} 2$ or $\mathrm{T} 3(15.0 \%)$. Data of the latter patients were included in the mixed models if values for the corresponding endpoints had been available. In the control group 211 patients (37.1\%) missed at least one follow-up measurement. Of these, 86 patients were completely lost to follow-up (15.1\%). Another 125 patients took part in at least one follow-up measurement so that their data were included in the mixed models $(22.0 \%)$. The number of patients lost to follow-up is illustrated in Figure 1 and the number of patients available for each analysis is listed in Table 1.

\section{Patient characteristics at baseline T0}

Patients' mean age was 63.8 years $( \pm 12.1)$ in the intervention group, and 65.0 years $( \pm 12.4)$ in the control group (cf. Table 2). In the intervention group $46.7 \%$ of patients were male, compared to $44.7 \%$ in the control group. At T0 $41.1 \%$ of the patients in the intervention group vs. $48.8 \%$ of the patients in the control group had at least one relevant comorbidity.

Perceived participation (SDM-Q-9) at T0 was relatively high both in the intervention group (mean score $73.00[ \pm 17.66]$ ) and in the control group (mean score 70.67 [ \pm 20.24$]$ ). Patients in both groups reported a very high medication adherence (MARS-D; mean score about 94 in both groups).

The 24h mean systolic / diastolic BP at T0 amounted to $133.2 / 82.0 \mathrm{mmHg}( \pm 13.6 / \pm 9.8)$ in the intervention group and $130.8 / 79.3 \mathrm{mmHg}( \pm 12.6 / \pm 9.7)$ in the control group. Almost $90.8 \%$ of the patients $(\mathrm{N}=501)$ in the intervention group and $84.7 \%$ of patients $(\mathrm{N}=481)$ in the control group exceeded at least one of the six ABPM thresholds ( $24 \mathrm{~h}$ or day or night), i.e. these patients were characterised as 'uncontrolled treated'. The $24 \mathrm{~h}$ mean systolic BP of patients with uncontrolled hypertension in the intervention group amounted to $134.9 \mathrm{mmHg}( \pm 12.8)$ and for the control group to $133.5 \mathrm{mmHg}( \pm 11.5)$. The $24 \mathrm{~h}$ mean systolic BP of all patients characterised as controlled hypertensive but with relevant comorbidity $(12.2 \%, \mathrm{~N}=138)$ was considerably lower and amounted to 


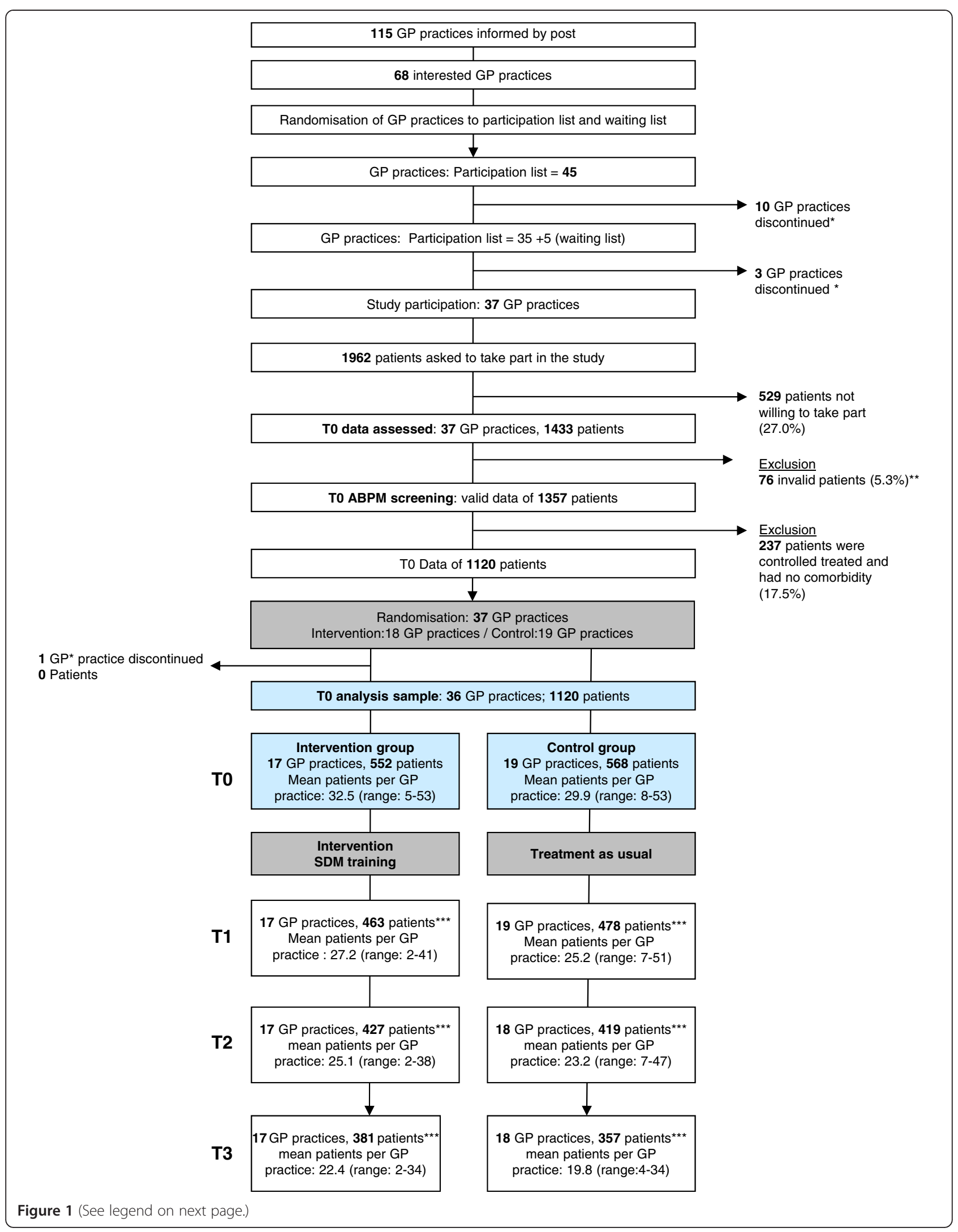


(See figure on previous page.)

Figure 1 Recruitment and study procedure including loss to follow-up. GP practices = general practitioner practices. * Details of discontinued GP practices: high work load and lack of staff. ** Details of invalid data: Enrolment in contrary to the inclusion criteria (patients were not member of a statutory health insurance or had not received repeated antihypertensive medication) or questionnaires were missing. ** Details of discontinued patients in both study arms: 1) Intervention group: 171 patients missed at least one follow-up (31.0\%). Thereof data of $\mathbf{8 8}$ patients are completely lost (15.9\%) (loss of T1, T2, and T3) and data of $\mathbf{8 3}$ patients (15.0\%) are partially available (loss of T1, T2 or T3). 2) Control group: $\mathbf{2 1 1}$ patients missed at least one follow-up (37.1\%; including 1 GP practice* with 3 active patients). Thereof data of $\mathbf{8 6}$ patients are completely lost (15.1\%) (loss of T1, T2, and T3) and data of $\mathbf{1 2 5}$ patients (22.0\%) are partially available (loss of T1, T2, or T3). Number of patients available for each analysis is listed in Table 1. Reasons for discontinuation of the patients: patients' desire $(N=241)$, change of $G P$ practice $(N=18)$, cardiovascular death

$(N=2)$, other death $(N=10)$, loss of GP practice (3 patients), other cause $(N=65)$, missing Documentation $(N=41)$.

$116.0 \mathrm{mmHg}( \pm 7.2)$ in the intervention group and to $115.8 \mathrm{mmHg}( \pm 6.7)$ in the control group.

After the first ABPM and the following adaptation of the therapy in $\mathrm{T} 0$, the mean $\mathrm{BP}$ values (systolic/diastolic) in the whole sample decreased by $3.4 / 2.4 \mathrm{mmHg}$ to
$127.9 / 78.0 \mathrm{mmHg}( \pm 12.2 / \pm 9.4)$ at T1. This decrease cannot result from the intervention because the intervention could have affected $\mathrm{BP}$ values after the T1 consultation at the earliest. The baseline BP value (T1) in the intervention group amounted to $128.9 / 79.2 \mathrm{mmHg}( \pm 12.5 / \pm 9.5)$ and in

Table 1 Mean changes of endpoints at each follow-up to baseline and differences in average mean changes (effect estimates)

\begin{tabular}{|c|c|c|c|c|c|c|c|c|c|}
\hline \multirow{2}{*}{$\begin{array}{l}\text { Mean changes } \\
\text { Number of GP practices }\end{array}$} & \multicolumn{3}{|c|}{ Intervention group } & \multicolumn{3}{|c|}{ Control group } & \multirow{2}{*}{$\begin{array}{c}\text { Difference in average } \\
\text { means change from T0/T1 } \\
\text { (intervention vs. control) }\end{array}$} & \multirow{2}{*}{$\begin{array}{l}\text { Confidence } \\
\text { interval }\end{array}$} & \multirow[t]{2}{*}{ Significance $p$} \\
\hline & 17 & 17 & 17 & 19 & 18 & 18 & & & \\
\hline \multicolumn{10}{|l|}{ Primary endpoints } \\
\hline SDM-Q-9 & T1-T0 & T2-T0 & T3-T0 & T1-T0 & T2-T0 & T3-T0 & T0 & & \\
\hline Valid N & 320 & 287 & 256 & 337 & 272 & 246 & & & \\
\hline Mean change & -0.11 & -2.875 & -1.07 & -4.06 & -2.34 & -3.69 & 3.1182 & $-2.3730 ; 8.6093$ & 0.2029 \\
\hline$(\mathrm{SD})$ & $(21.45)$ & $(22.98)$ & $(21.50)$ & $(22.51)$ & $(20.73)$ & $(20.87)$ & & & \\
\hline Systolic BP & & T2-T1 & T3-T1 & & T2-T1 & T3-T1 & $\mathrm{T} 1$ & & \\
\hline Valid N & & 414 & 381 & & 376 & 337 & & & \\
\hline Mean change in $\mathrm{mmHg}$ & & 0.59 & 0.43 & & -0.02 & -0.81 & 1.7517 & $-0.1884 ; 3.6918$ & 0.0430 \\
\hline$(\mathrm{SD})$ & & $(11.54)$ & (12.08) & & $(10.60)$ & $(10.92)$ & & & \\
\hline \multicolumn{10}{|l|}{ Secondary endpoints } \\
\hline Diastolic BP & & T2-T1 & T3-T1 & & T2-T1 & T3-T1 & $\mathrm{T} 1$ & & \\
\hline Valid N & & 414 & 381 & & 376 & 337 & & & \\
\hline Mean change in $\mathrm{mmHg}$ & & -0.20 & -0.15 & & -0.15 & -0.66 & 0.9377 & $-0.0381 ; 1.9134$ & 0.0596 \\
\hline$(\mathrm{SD})$ & & $(6.74)$ & $(6.14)$ & & $(6.14)$ & $(5.86)$ & & & \\
\hline CVR & & T2-T1 & T3-T1 & & T2-T1 & T3-T1 & $\mathrm{T} 1$ & & \\
\hline Valid N & & 294 & 282 & & 255 & 212 & & & \\
\hline Mean change in \% & & 0.71 & 1.44 & & 1.14 & 2.04 & -0.4891 & $-1.4307 ; 0.4526$ & 0.3084 \\
\hline$(\mathrm{SD})$ & & $(5.43)$ & $(6.89)$ & & $(5.58)$ & $(8.50)$ & & & \\
\hline MARS-D & & T2-T1 & T3-T1 & & T2-T1 & T3-T1 & $\mathrm{T} 1$ & & \\
\hline Valid N & & 360 & 320 & & 313 & 287 & & & \\
\hline Mean change & & 0.07 & 0.4 & & -0.3 & -1.1 & 0.670 & $-0.3748 ; 1.7166$ & 0.2084 \\
\hline$(\mathrm{SD})$ & & $(9.6)$ & $(10.7)$ & & $(6.9)$ & $(6.7)$ & & & \\
\hline Knowledge & T1-T0 & T2-T0 & T3-T0 & T1-T0 & T2-T0 & T3-T0 & T0 & & \\
\hline Valid N & 446 & 402 & 363 & 428 & 369 & 330 & & & \\
\hline Mean change & 3.00 & 2.99 & 5.85 & 3.30 & 3.76 & 4.66 & 1.3267 & $-4.3272 ; 6.9806$ & 0.6454 \\
\hline (SD) & $(27.71)$ & $(28.56)$ & $(28.44)$ & $(30.00)$ & $(28.37)$ & $(24.49)$ & & & \\
\hline
\end{tabular}


Table 2 Patient characteristics at T0

\begin{tabular}{|c|c|c|}
\hline Characteristics at T0 & $\frac{\text { Intervention group }}{(\mathrm{N}=552)}$ & $\frac{\text { Control group }}{(\mathrm{N}=568)}$ \\
\hline Age mean (SD) & $63.8( \pm 12.1)$ & $65.0( \pm 12.4)$ \\
\hline Gender: male \% (N) & $46.7 \%(258)$ & $44.7 \%(254)$ \\
\hline Educational level (Total valid N) & $(523)$ & $(521)$ \\
\hline low & $62.7 \%(328)$ & $63.8 \%(332)$ \\
\hline middle & $24.1 \%(126)$ & $22.6 \%(118)$ \\
\hline high & $13.2 \%(69)$ & $13.6 \%(71)$ \\
\hline ABDM (Total valid N) & (552) & (568) \\
\hline 24h-mean systolic (SD) & $133.19(13.58)$ & $130.80(12.62)$ \\
\hline 24h-mean diastolic (SD) & $81.98(9.77)$ & $79.29(9.74)$ \\
\hline Uncontrolled hypertension & $90.8 \%(501)$ & $84.7 \%(481)$ \\
\hline$\geq 1$ Comorbidity & $41.1 \%(227)$ & $48.8 \%(277)$ \\
\hline Diabetes mellitus & $23.7 \%(131)$ & $32.6 \%(185)$ \\
\hline Heart attack / CHD & $16.8 \%(93)$ & $20.8 \%(118)$ \\
\hline Stroke/ TIA & $4.7 \%(26)$ & $5.5 \%(31)$ \\
\hline Peripheral arterial occlusive disease & $3.6 \%(20)$ & $4.1 \%(23)$ \\
\hline Manifest arteriosclerosis (heart attack, CHD, stroke, TIA, or PAOD) & $22.6 \%(125)$ & $26.4 \%(150)$ \\
\hline CVR score in \% (Total valid N) & $(420)$ & $(377)$ \\
\hline Mean (SD) & $20.40( \pm 19.1)$ & $22.85( \pm 18.71)$ \\
\hline BMI (Total valid N) & $(545)$ & $(561)$ \\
\hline Mean (SD) & $28.58( \pm 4.78)$ & $28.58( \pm 4.79)$ \\
\hline Smoking (Total valid N) & $(544)$ & (558) \\
\hline Positive (N) & $11.2 \%(861)$ & $11.6 \%(65)$ \\
\hline Family history of CVD (Total valid N) & (528) & $(527)$ \\
\hline Positive (N) & $24.2 \%(128)$ & $19.4 \%(102)$ \\
\hline Treatment outside the GP praxis & $35.3 \%(195)$ & $35.0 \%(199)$ \\
\hline SDM-Q-9* (Total valid N) & $(451)$ & (489) \\
\hline Mean (SD) & $73.00( \pm 17.66)$ & $70.67( \pm 20.24)$ \\
\hline Intention-to-treat-hypertension scale ${ }^{*}($ Total valid N) & $(521)$ & $(527)$ \\
\hline Mean (SD) & $80.61( \pm 13.32)$ & $79.79( \pm 13.52)$ \\
\hline MARS-D*(Total valid N) & $(531)$ & (539) \\
\hline Mean (SD) & $93.9( \pm 9.8)$ & $93.5( \pm 10.1)$ \\
\hline Knowledge $^{*}$ (Total valid N) & (536) & $(547)$ \\
\hline Mean (SD) & $46.63( \pm 27.60)$ & $44.22( \pm 28.64)$ \\
\hline
\end{tabular}

* Scale $0-100(0=$ lowest level and $100=$ highest level).

ABPM ambulatory blood pressure monitoring, CHD coronary heart disease, TIA transient ischemic attack, PAOD peripheral arterial occlusive disease, CVR cardiovascular risk, BMI Body Maas Index, CVD, cardiovascular disease, SDM-Q-9 Shared Decision Making-Questionnaire, MARS-D Medication Adherence Report Scale-German Version.

the control group to $127.0 / 76.8 \mathrm{mmHg}[ \pm 11.8 / \pm 9.1)$ (cf. Table 3). Even though the mean BP values in both study arms were below recommended BP values for ABPM in T1, the proportion of patients with uncontrolled (but treated) hypertension (as defined: at least one of the six ABPM thresholds was exceeded) in the intervention group amounted to $76.9 \%(\mathrm{~N}=356)$ and in the control group to $71.6 \%(\mathrm{~N}=327)$ (cf. Table 4).

\section{Effects of the SDM training on primary endpoints} Effect on patients' perceived participation (SDM-Q-9)

At baseline assessment (T0), the intervention group had a slightly higher mean SDM-Q-9 score than the control group (cf. Figure 2). The mean SDM-Q-9 score in the control group decreased after baseline assessment. The patients of the control group perceived the highest participation after the first consultation reviewing the 
Table 3 Patient characteristics at each assessment point

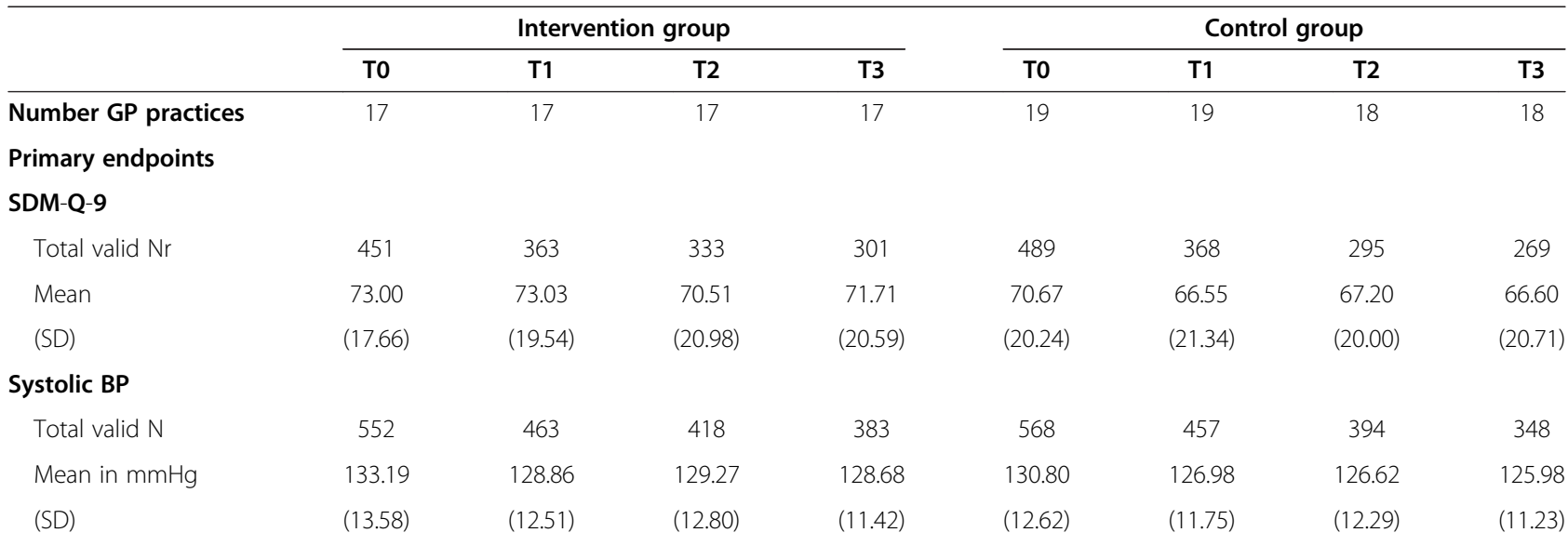

Secondary endpoints

\section{Diastolic BP}

$\begin{array}{lcccccccc}\text { Total valid N } & 552 & 463 & 418 & 383 & 568 & 457 & 394 & 348 \\ \text { Mean in } \mathrm{mmHg} & 81.98 & 79.21 & 79.07 & 78.55 & 79.29 & 76.75 & 76.75 & 75.64 \\ \text { (SD) } & (9.77) & (9.49) & (8.79) & (8.81) & (9.74) & (9.10) & (9.10) & (8.46)\end{array}$

\section{CVR}

$\begin{array}{lcccccccc}\text { Total valid N } & 420 & 364 & 320 & 312 & 377 & 336 & 270 & 233 \\ \text { Mean in \% } & 20.40 & 21.02 & 22.33 & 23.92 & 22.85 & 23.54 & 24.03 & 24.34 \\ \text { (SD) } & (18.15) & (18.61) & (18.82) & (19.10) & (18.71) & (19.19) & (19.23) & (19.60)\end{array}$

MARS-D

\begin{tabular}{lcccccccc} 
Total valid N & 531 & 419 & 387 & 349 & 539 & 408 & 357 & 326 \\
Mean & 93.9 & 95.4 & 96.0 & 96.1 & $93.5)$ & 96.0 & 96.1 & 95.3 \\
(SD) & $(9.8)$ & $(8.8)$ & $(6.8)$ & $(8.0)$ & $(10.1)$ & $(6.3)$ & $(6.8)$ & $(7.5)$ \\
Knowledge & 536 & 455 & 412 & 371 & 547 & 444 & 382 & 343 \\
Total valid N & 536 & 455 & 412 & 371 & 547 & 444 & 382 & 343 \\
Mean & 46.63 & 50.05 & 50.18 & 53.34 & 44.22 & 47.04 & 47.64 & 48.51 \\
(SD) & $(27.60)$ & $(28.39)$ & $(28.32)$ & $(28.21)$ & $(28.64)$ & $(30.11)$ & $(30.49)$ & $(30.15)$ \\
\hline
\end{tabular}

BP Blood Pressure, SDM-Q-9 Shared Decision Making-Questionnaire, CVR cardiovascular risk, MARS-D Medication Adherence Report Scale-German Version.

Table 4 Patients with uncontrolled (but treated) blood pressure from T0 to T3

\begin{tabular}{lcc}
\hline Uncontrolled BP*: percentage & Intervention group & Control group \\
\hline T0 Total valid numbers N & 552 & 568 \\
Uncontrolled BP Valid percentages \% (number N) & $90.8 \%(501)$ & $84.7 \%(481)$ \\
T1 Total valid numbers N & 463 & 457 \\
Uncontrolled BP Valid percentages \% (number N) & $76.9 \%(356)$ & $71.6 \%(327)$ \\
T2 Total valid numbers N & 418 & 394 \\
Uncontrolled BP Valid percentages \% (number N) & $78.2 \%(327)$ & $39.0 \%(272)$ \\
T3 Total valid numbers N & 383 & 348 \\
Uncontrolled BP Valid percentages \% (number N) & $78.3 \%(300)$ & $69.8 \%(243)$ \\
\hline
\end{tabular}

*Uncontrolled BP: if $24 \mathrm{~h}$-mean $\geq 130 / 80 \mathrm{mmHg}$ or day-mean $\geq 135 / 85 \mathrm{mmHg}$ or night-mean $\geq 120 / 70 \mathrm{mmHg}$. 


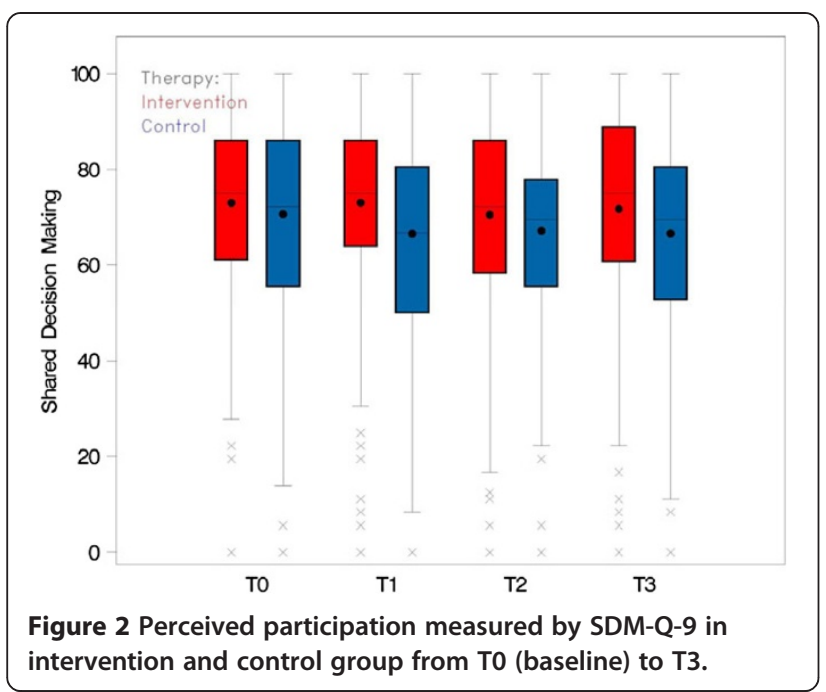

ABPM protocol. In the intervention group the mean SDM-Q-9 score remained approximately on the same level at T1 but it also decreased slightly after T1 (cf. Figure 3). After the intervention, the average decrease of the SDM-Q-9 score was slightly smaller in the intervention group than in the control group. According to the mixed model analysis, the average change from T0 was 3.11 points higher in the intervention group than in the control group (97.5\% CI [-2.37; 8.61], $\mathrm{p}=0.203)$. The effect was not significant at the (Bonferroni-corrected) 2.5\% level (cf. Table 1).

\section{Effect on systolic blood pressure}

In all four data assessments the mean systolic BP was slightly higher in the intervention group than in the control group (cf. Figure 4 and Table 3). Distinct decreases in BP could be observed in both study arms between T0 and $\mathrm{T} 1$, i.e. after the initial assessment, and a possible change in patient's treatment (cf. Figure 4). BP

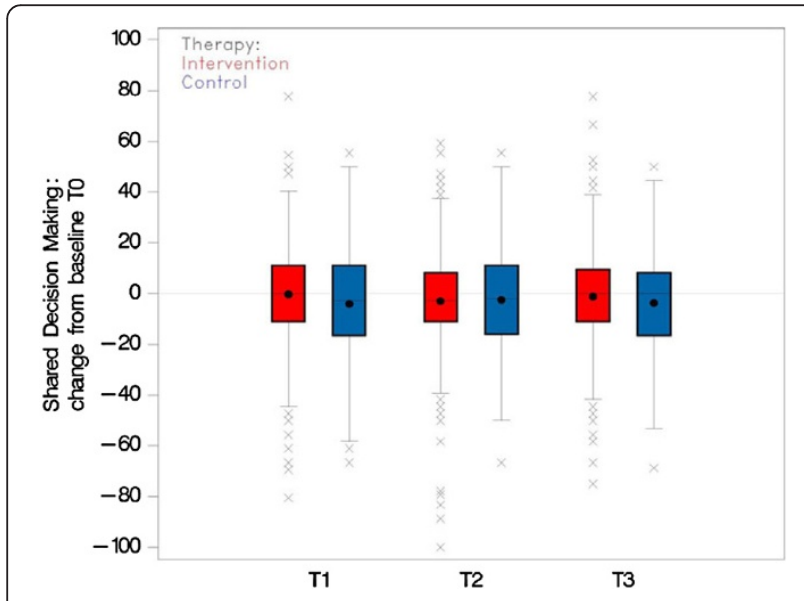

Figure 3 Average change of SDM-Q-9 from T1, T2, and T3 relative to T0 (baseline) in intervention and control group.

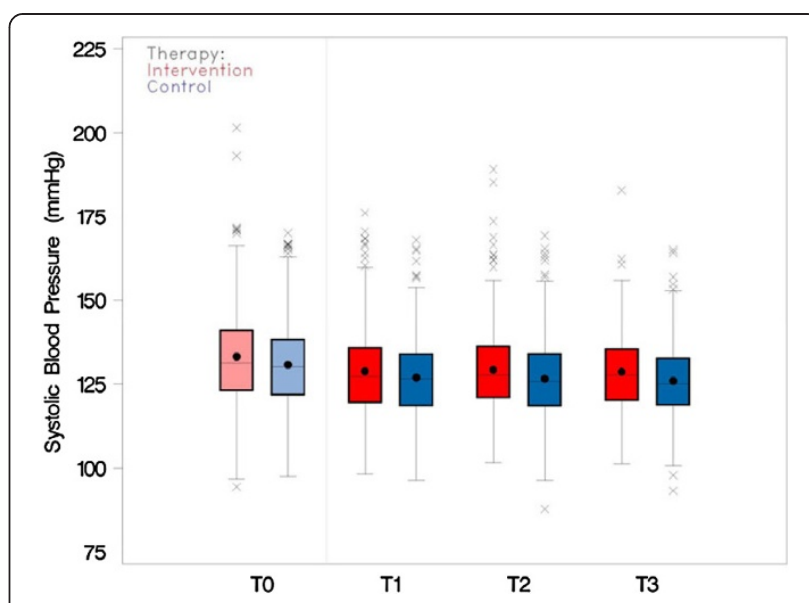

Figure 4 Systolic blood pressure from T0 (screening value), and T1 (baseline) to T3 in intervention and control group.

measurements at T2 and T3, which were expected to be affected by the SDM training, did not show relevant changes compared to T1 (cf. Figure 5). The average change in BP from $\mathrm{T} 1$ was - according to the mixed model analysis - higher in the intervention group than in the control group (effect estimate +1.75 $\mathrm{mmHg}(97.5 \% \mathrm{CI}[-0.189 ; 3.69], \mathrm{p}=0.043)$. This resulted from a slight BP decrease in the control group and a slight $\mathrm{BP}$ increase in the intervention group (cf. Table 1). The comparative increase of systolic BP in the intervention group was against our expectations. However, it is not significant at the level of $2.5 \%$. The sensitivity analyses with (1) baseline T0 and (2) 12 additional prognostic factors showed similar trends with average intervention effects of $+1.07 \mathrm{mmHg}(97.5 \% \mathrm{CI}[0.76 ; 2.90])$ and $+2.39 \mathrm{mmHg}(97.5 \% \mathrm{CI}[0.23 ; 4.53])$, respectively.

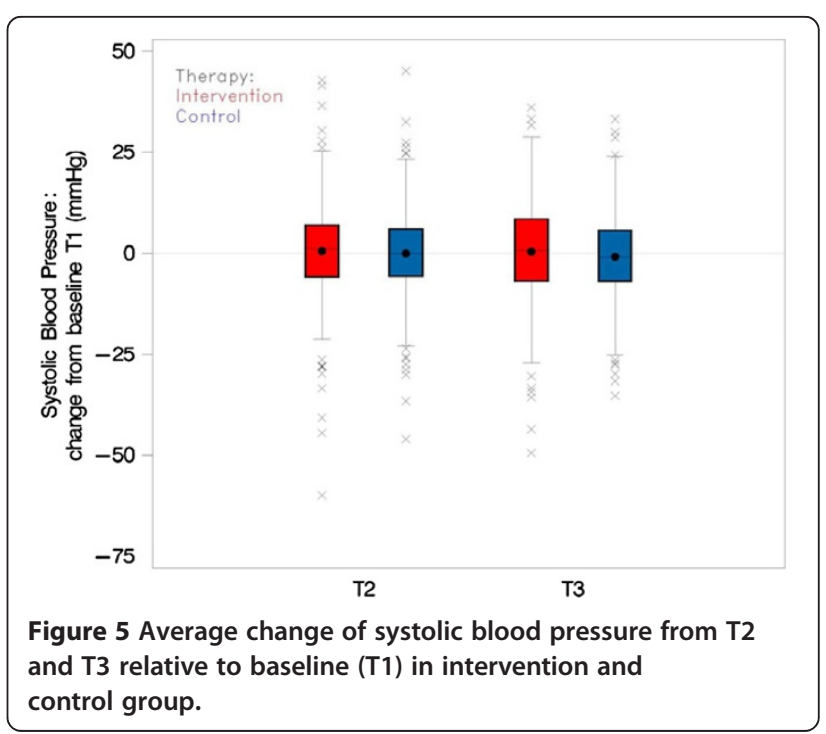


In summary, the analyses showed unexpected trends: (1) the positive (but not significant) intervention effect on participation resulted from a decreased participation in the control group rather than an increase in the intervention group. And (2) a comparative (but not significant) increase of systolic blood pressure was observed in the intervention group compared to the control group.

\section{Effects of the SDM training on secondary endpoints}

The mean changes in secondary endpoint variables were very small, and there was no significant intervention effect on any of these endpoints (at significance level 5\%). The intervention led to a negligible decrease of $0.49 \%$ in CVR (95\% CI [-1.43; 0.45], p = 0.308) in the intervention group compared to the control group. The knowledge of the patients about hypertension increased slightly in both study arms. However, the mean intervention effect amounted to only +1.33 points (95\% CI [-4.33; 6.98], $\mathrm{p}=0.645)$ and was not significant. The mean adherence score (MARS-D) was very high at the beginning of the study (cf. Table 3), thus leaving little potential for improvement. The mean intervention effect (cf. Table 1) amounted to only 0.67 points (95\% CI $[-0.37 ; 1.72]$, $\mathrm{p}=0.208)$.

\section{Discussion}

\section{Main results}

The results of this study showed that the SDM training programme for GPs neither enhanced patients' participation significantly nor contributed to a BP decrease, to a higher adherence or knowledge. The CVR-lowering effect of the SDM training amounted to $0.49 \%(\mathrm{p}=0.308)$, was negligible and not significant. Deviating from our hypothesis the SDM training did not show any relevant effect on primary or secondary endpoints. Instead, the implementation of the ABPM initiated an improvement of the hypertension treatment, followed by a mean BP decrease between $\mathrm{T} 0$ and $\mathrm{T} 1$ by (systolic/diastolic) $3.4 / 4.4 \mathrm{mmHg}$ $( \pm 12.2 / \pm 9.4)$ in all patients.

\section{Strengths and weaknesses of the study}

Because most of the participating GPs were academic family doctors associated with the Division of General Practice of the University Medical Centre Freiburg (Germany), the external validity of our results seems to be limited. Therefore we may assume that the participating GPs are probably more open-minded as to taking part in studies and improving their skills when compared to all GPs in the region. Thus, e.g., we assume that GPs who were interested in improving their antihypertensive treatment or their communication skills were overrepresented in this study.

The internal validity of the study is limited to some extent because the intervention itself could not be blinded for the GPs. With respect to the endpoint 'patients' perceived participation' our study might also be internally limited: Recent discussions about the SDM-Q-9 instrument have raised doubts about its content validity $[48,49]$. Nevertheless the internal validity of the data seems to be fairly high. The loss to follow-up in primary care studies with long term follow up is higher than in clinical studies and was taken into consideration in our sample size calculation. In both arms of this study about $15 \%$ of the patients were completely lost to followup $(15.9 \%$ in the intervention group and $15.1 \%$ in the control group). Another $15 \%$ in the intervention group and $22 \%$ in the control group were lost at one or two of the three follow-ups. In the power calculation we estimated that 788 patients would finish the T3 assessment and this number was nearly achieved $(\mathrm{N}=738)$. In total the missing patterns were rather similar in both study arms. Additionally, the implementation of a cluster-randomised design and a rigorous statistical analysis reinforce the evidence of the results. Therefore the results of our study seem sufficiently valid.

It would have been desirable to evaluate to what extent the GPs in the intervention group really implemented a communication style in accordance with the SDM principles. As we measured only patients' perceptions on the subject, we do not know to what extent the GPs of the intervention group changed their behaviour 'objectively'.

\section{Comparison with other studies and interpretation}

In comparison with other studies in which an SDM intervention had been effective $[15,17,24]$ our GP training was rather short. Reviews investigating the effects of SDM interventions ascertain that it is often difficult to bring about behavioural change in doctors with short training programmes $[20,21]$. Thus one could argue that a more intensive training or other dissemination strategies like coaching on the job or internet-driven feedback strategies might be useful to change GPs' communication behaviour. However in the study by Deinzer et al. [25], which was characterised by an SDM programme for GPs with a $16 \mathrm{~h}$ communication training plus supervision, the intervention neither enhanced participation nor improved BP control. A Cochrane review from 2010 analysed various educational training programmes (not confined to SDM training) directed to physicians to improve antihypertensive treatment and did not find any effective training which significantly lowered patients' BP [50]. A recent review concluded that to date there were no consistent results regarding the effectiveness of patient participation on health-related outcomes [51].

From prior process evaluations of our SDM training we may presume that the intervention was sufficiently accepted by the participating GPs. An intensive and 'invasive' training or a direct evaluation of consultations, however, would 
probably have affected GPs' acceptance of the intervention and the feasibility of the study.

Our finding that BP decreased between T0 and T1 is in line with other studies which showed that the implementation of ABPM contributed to a more effective antihypertensive treatment [52-54]. However, it is necessary to take into consideration that at $\mathrm{T} 0$ the mean $24 \mathrm{~h} \mathrm{BP}$ values of the whole sample only marginally exceeded the recommended ABPM thresholds. Moreover, the consultation that immediately followed the ABPM at T0 provided the opportunity to GPs and patients to optimise their antihypertensive therapy with all usual treatment options in both study arms before the SDM training for GPs started. Accordingly, in T1 the mean 24h BP values were even below the ABPM diagnostic thresholds in both study arms. This might indicate that systolic or diastolic BP values of most patients whose hypertension was characterised as treated but uncontrolled in $\mathrm{T} 1$ (intervention group: $76.9 \%[\mathrm{~N}=356]$, control group: $71.6 \%$ [ $\mathrm{N}=327]$, cf. Table 4) probably exceeded the recommended ABPM thresholds - e.g. at night - only marginally. Taking into account these circumstances, aggressive treatment escalations could not be expected for the majority of the study population after T1. Therefore one could expect only relatively minor treatment responses to further treatment changes on mean BP.

\section{Conclusion}

Considering that a distinct BP decrease could be noticed in both study groups at the very beginning of the study, i.e. between T0 and T1, we may put forward the hypothesis that the implementation of ABPM can lead to a therapeutic adaption with a positive effect on BP optimisation. The subsequent implementation of a rather short SDM training for GPs does obviously not increase the effectiveness of such a therapeutic adaptation regarding BP decrease.

Though well accepted by the GPs, our results seem to discourage the optimistic expectation that SDM training for GPs solely leads to a relevant increase in patients' perceived participation or an improvement of clinical outcomes. As patient participation is established in law in Germany, further research should be undertaken to examine the process of patient participation in primary care.

\section{Abbreviations}

ABPM: Ambulatory blood pressure monitoring/measurement; BP: Blood pressure; CBPM: Clinical blood pressure measurement; CHD: Coronary heart disease; CRCT: Cluster randomised controlled trial; CVD: Cardiovascular disease; CVR: Cardiovascular risk; DFG: German Research Foundation; EMA: European medicine agency; GP: General practitioners; ICC: Intra-cluster correlation; ITT: Intention-to-treat; MARS-D: Medication adherence report scale - German version; PAOD: Peripheral arterial occlusive disease; SAS: Statistical analysis system; SDM: Shared decision making; SDM-Q-9: 9-item-questionnaire measuring patients' perceived participation in medical decision making; TIA: Transient ischaemic attack.
}

\section{Competing interests}

The authors declare that they have no competing interests.

\section{Authors' contributions}

KGF, WN, TD, IT conceived the study. IT was responsible for the realisation of the project and its data management. $A B$ and $W$ carried out the mixed model analyses. AS, TD, IT, AB, and $W$ interpreted the results. All authors were involved in drafting the manuscript and revised it critically. All authors read and approved the final manuscript.

\section{Authors' information}

$\mathrm{IT}^{1}$ : Sociologist (M.A.), coordinator of the study.

$A B^{2}$ : Statistician (diploma and PhD). Biostatistician at the Clinical Trials Unit

and the Institute of Medical Biometry and Medical Informatics at the University Medical Centre Freiburg.

$\mathrm{W}^{3}$ : Statistician (diploma and PhD) and Professor of medical informatics and clinical epidemiology at the University of Freiburg.

$\mathrm{AS}^{1}$ : Social scientist (PhD and MPH).

$\mathrm{TD}^{1}$ : Assistant physician, $\mathrm{MD}$ (Dr. med.) and researcher.

WN ${ }^{1}$ : General practitioner, MD (Dr. med). Professor and head of the Division of General Practice at the University Medical Centre Freiburg, Germany. Board member of the commission of the German Agency for Quality in

Medicine (AQuMed/AEZQ), decision board member of the German Medical Association and co-editor of the German Journal of Family Medicine (ZFA). $A B^{4}$ : Psychologist (diploma and PhD), senior researcher.

$\mathrm{KGF}^{5}$ : Nephrologist, MD (Dr. med). Working with the Division of Nephrology, University Medical Centre Freiburg, and a dialysis centre in Lörrach, Germany.

\section{Acknowledgement}

This study was funded by the German Federal Ministry of Education and Research (Grant number 01GX0731). This funding included the acquisition and implementation of the ABPM equipment in all GP practices. The article processing charge in BMC Family Practice was funded by the German Research Foundation (DFG) and the Albert Ludwig University of Freiburg in the funding programme Open Access Publishing. First and foremost we would like to thank all patients, GPs and the GP practice staff for taking part in this study. Thanks to Dr. Andreas Loh and Dr. Sabine Beck; both were substantially involved in the planning of the study. Many thanks to our study nurse Angela Kotterer and all doctoral students Michael Bösch, Mareike Füller, Caroline Bollmann, Anke Pfaff, Mathias Sauler, Titia Schardt and Stephanie Spiegler for their support in the data entry. The Department of General Practice and Health Services Research and Department of Internal Medicine VI, Clinical Pharmacology and Pharmacoepidemiology, University Hospital Heidelberg, Germany, provided the MARS-D. Prof. Norbert DonnerBanzhoff (Marburg) and Prof. Attila Altiner (Rostock) project leaders of the cardiovascular risk calculator (arriba) provided the CVR algorithm for our analyses; special thanks to Thomas Scheithauer for technical support. Thanks to Dr. Klaus Böhme, Department of Medicine, Division of General Practice, University Medical Centre Freiburg, for his excellent medical support and to Prof. Michael M. Kochen (Freiburg) for reviewing the manuscript.

\section{Author details}

${ }^{1}$ Department of Medicine, Division of General Practice, University Medical Centre Freiburg, Elsässerstr 2m, Freiburg 79110, Germany. ${ }^{2}$ Clinical Trials Unit, University Medical Centre Freiburg, Elsässerstr 2, Freiburg 79110, Germany. ${ }^{3}$ Institute of Medical Biometry and Medical Informatics, University Medical Centre Freiburg, Stefan-Meier-Str. 26, Freiburg 79104, Germany. ${ }^{4}$ Department of Medical Psychology, University Medical Centre Hamburg-Eppendorf, Martinistraße 52, Hamburg 20246, Germany. ${ }^{5}$ Department of Medicine, Division of Nephrology, University Medical Centre Freiburg, Hugstetterstr. 55, Freiburg 79106, Germany.

Received: 10 April 2013 Accepted: 20 August 2013 Published: 11 September 2013

\section{References}

1. U.S. Department of Health and Human Services, National Institutes of Health, National Heart Lung and Blood Institute: The seventh report of the joint national committee on prevention, detection, evaluation, and treatment of high blood pressure. Full version. NIH Publication No 04-5230 2004:1-88. [http://www.nhlbi.nih.gov/guidelines/hypertension/jnc7full.pdf] (last accessed September 05, 2013)

2. Robert Koch Institute: Daten und Fakten: Ergebnisse der Studie "Gesundheit in Deutschland aktuell 2009" [Data and facts: Results of the 
study "Health in Germany 2009"]. In Gesundheitsberichterstattung des Bundes [Federal Health Monitoring System]. Berlin; 2009:1-139. [in German, English abstract not available] http://www.gbe-bund.de/gbe10/owards. prc_show_pdf?p_id=13126\&p_sprache=D (last accessed August 02, 2013).

3. Ostchega $Y$, Dillon CF, Hughes JP, Carroll M, Yoon S: Trends in hypertension prevalence, awareness, treatment, and control in older U.S. adults: data from the National Health and Nutrition Examination Survey 1988 to 2004. J Am Geriatr Soc 2007, 55(7):1056-1065.

4. Egan BM, Zhao Y, Axon RN, Brzezinski WA, Ferdinand KC: Uncontrolled and apparent treatment resistant hypertension in the United States, 1988 to 2008. Circulation 2011, 124(9):1046-1058.

5. Valderrama AL, Tong X, Ayala C, Keenan NL: Prevalence of self-reported hypertension, advice received from health care professionals, and actions taken to reduce blood pressure among US adults-HealthStyles, 2008. J Clin Hypertens 2010, 12(10):784-792.

6. Khanna RR, Victor RG, Bibbins-Domingo K, Shapiro MF, Pletcher MJ: Missed Opportunities for Treatment of Uncontrolled Hypertension at Physician Office Visits in the United States, 2005 Through 2009. Arch Intern Med 2012, 172(17):1344-1345.

7. Leenen FH, Dumais J, Mclnnis NH, Turton P, Stratychuk L, Nemeth K, Lum-Kwong MM, Fodor G: Results of the Ontario survey on the prevalence and control of hypertension. CMAJ 2008, 178(11):1441-1449.

8. Luepker RV, Steffen LM, Jacobs DR Jr, Zhou X, Blackburn H: Trends in blood pressure and hypertension detection, treatment, and control 1980 to 2009: the Minnesota Heart Survey. Circulation 2012, 126(15):1852-1857.

9. Brenner R, Waeber B, Allemann Y: Medical treatment of hypertension in Switzerland. The 2009 Swiss Hypertension Survey (SWISSHYPE). Swiss Med Wkly 2011, 141:W13169 (E1-E8).

10. Meisinger C, Heier M, Volzke H, Lowel H, Mitusch R, Hense HW, Ludemann $\mathrm{J}$ : Regional disparities of hypertension prevalence and management within Germany. J Hypertens 2006, 24(2):293-299.

11. Wang YR, Alexander GC, Stafford RS: Outpatient hypertension treatment, treatment intensification, and control in Western Europe and the United States. Arch Intern Med 2007, 167(2):141-147.

12. Janhsen $K$, Strube H, Starker A: Hypertonie [Hypertension]. In Gesundheitsberichtserstatung des Bundes [Federal Health Monitoring System]. Berlin; 2008:1-38. [in German, English abstract not available] http://www.gbe-bund.de/gbe10/owards.prc show_pdf? p_id $=11737 \&$ p_sprache $=d \& p \_u i d=g a s t g \& p \_a i d=32760807 \& p \_l f d \_n r=1$ (last accessed July 30, 2013).

13. Federal Ministry of Health, Federal Ministry of Justice: Patientenrechte in Deutschland. Leitfaden für Patientinnen/Patienten und Ärztinnen/Ärzte [Patients' rights in Germany - guideline for patients and physicians in Germany]. Berlin: The Federal Ministry of Health; 2007:1-22. [in German, no English abstract available] http://www.berlin.de/imperia/md/content/lbpatienten/broschuere_patientenrechte_deutschland_0907.pdf? start\&ts=1201082859\&file=broschuere_patientenrechte_deutschland_0907. pdf (last accessed July 30, 2013)

14. Parliament of the Federal Republic of Germany: Verbesserung der Rechte von Patientinnen und Patienten[Improving the rights of patients]. Berlin: Bundesanzeiger Verlag. Part I Nr 9, Berlin, 25. Februar 2013:277-282 [in German, no English abstract available] [http://www.bgbl.de/Xaver/start.xav? startbk=Bundesanzeiger BGBI\&jumpTo=bgbl113s0277.

pdf\#_Bundesanzeiger_BGBI_\%2F\%2F*\%5B\%40attr_id\%3D'bgbl113s0277. pdf'\%5D_1375175860785] (last accessed July 29, 2013).

15. Loh A, Leonhart R, Wills CE, Simon D, Harter M: The impact of patient participation on adherence and clinical outcome in primary care of depression. Patient Educ Couns 2007, 65(1):69-78.

16. Legare F, Labrecque M, Cauchon M, Castel J, Turcotte S, Grimshaw J: Training family physicians in shared decision-making to reduce the overuse of antibiotics in acute respiratory infections: a cluster randomized trial. CMAJ 2012, 184(13):E726-E734

17. Bieber C, Muller KG, Blumenstiel K, Schneider A, Richter A, Wilke S, Hartmann M, Eich W: Long-term effects of a shared decision-making intervention on physician-patient interaction and outcome in fibromyalgia. A qualitative and quantitative 1 year follow-up of a randomized controlled trial. Patient Educ Couns 2006, 63(3):357-366

18. National Clinical Guideline Centre: Hypertension. The clinical management of primary hypertension in adults. Clinical Guideline 127. London: Commissioned by National Institute for Health and Clinical Excellence
(NICE); 2011:1-325. [http://www.nice.org.uk/nicemedia/live/13561/56007/ 56007.pdf] (last accessed September 13, 2103).

19. Bergert FW, Braun M, Clarius H, Ehrenthal K, Feßler J, Gross J, Hintze J, Hüttner U, Kluthe B, Liesenfeld A, Luther E, Seffrin J, Vetter G, Popert U, Ludt S, Schubert I: In Hausärztliche Leitlinie kardiovaskuläre Prävention [Primary care guideline for cardiovascular prevention]. Edited by Guideline Group Hessen. Version 1.00; 2011:1-106 [in German, no English abstract available]. http://www.pmvforschungsgruppe.de/pdf/03_publikationen/ kardiopraev_Il.pdf (last accessed July 29, 2013).

20. Legaré F, Ratté S, Stacey D, Kryworuchko J, Gravel K, Graham ID, Turcotte S: Interventions for improving the adoption of shared decision making by healthcare professionals. Cochrane Database Syst Rev 2010, 5, CD006732.

21. Legare F, Turcotte S, Stacey D, Ratte S, Kryworuchko J, Graham ID: Patients' perceptions of sharing in decisions: a systematic review of interventions to enhance shared decision making in routine clinical practice. The Patient 2012, 5(1):19.

22. Loh A, Simon D, Wills CE, Kriston L, Niebling W, Härter M: The effects of a shared decision-making intervention in primary care of depression: a cluster-randomized controlled trial. Patient Educ Couns 2007 67(3):324-332

23. Legare F, Politi MC, Drolet R, Desroches S, Stacey D, Bekker $H$, the SDM-CPD team: Training health professionals in shared decision-making: an international environmental scan. Patient Educ Couns 2012, 88(2):159-169.

24. Krones T, Keller H, Sonnichsen A, Sadowski EM, Baum E, Wegscheider K, Rochon J, Donner-Banzhoff N: Absolute cardiovascular disease risk and shared decision making in primary care: a randomized controlled trial. Ann Fam Med 2008, 6(3):218-227.

25. Deinzer A, Veelken R, Kohnen R, Schmieder RE: Is a shared decision-making approach effective in improving hypertension management? J Clin Hypertens 2009, 11(5):266-270.

26. Bieber C, Loh A, Ringel N, Eich W, Härter M: Patientenbeteiligung bei medizinischen Entscheidungen - Manual zur Partizipativen Entscheidungsfindung (Shared Decision-Making) [Patient participation in medical desicons - manual for shared decision-making]. Heidelberg: Heidelberg University Hospital (author's edition); 2007 [In German, no English abstract available].

27. European Medicines Agency (EMA): Guideline on clinical investigation of medicinal products in the treatment of hypertension. London: EMA/238/1995/ Rev; 2010:1-18.

28. Mancia G, De Backer G, Dominiczak A, Cifkova R, Fagard R, Germano G, Grassi G, Heagerty AM, Kjeldsen SE, Laurent S, Narkiewicz K, Ruilope L, Rynkiewicz A, Schmieder RE, Boudier HA, Zanchetti A, Vahanian A, Camm J, De Caterina R, Dean V, Dickstein K, Filippatos G, Funck-Brentano C, Hellemans I, Kristensen SD, McGregor K, Sechtem U, Silber S, Tendera M, Widimsky P, et al: Guidelines for the management of arterial hypertension: the task force for the management of arterial hypertension of the European Society of Hypertension (ESH) and of the European Society of Cardiology (ESC). J Hypertens 2007, 25(6):1105-1187.

29. Franssen PM, Imholz BP: Evaluation of the Mobil-O-Graph new generation ABPM device using the ESH criteria. Blood Press Monit 2010, 15(4):229-231.

30. Wei W, Tolle M, Zidek W, van der Giet M: Validation of the mobil-O-Graph: 24 h-blood pressure measurement device. Blood Press Monit 2010, 15(4):225-228

31. O'Brien E, Asmar R, Beilin L, Imai Y, Mancia G, Mengden T, Myers M, Padfield P, Palatini P, Parati G, Pickering T, Redon J, Staessen J, Stergiou G, Verdecchia P: Practice guidelines of the European Society of Hypertension for clinic, ambulatory and self blood pressure measurement. J Hypertens 2005, 23(4):697-701.

32. Kriston L, Scholl I, Holzel L, Simon D, Loh A, Härter M: The 9-item Shared Decision Making Questionnaire (SDM-Q-9). Development and psychometric properties in a primary care sample. Patient Educ Couns 2010, 80(1):94-99.

33. Mahler C, Hermann K, Horne R, Ludt S, Haefeli WE, Szecsenyi J, Jank S: Assessing reported adherence to pharmacological treatment recommendations. Translation and evaluation of the Medication Adherence Report Scale (MARS) in Germany. J Eval Clin Pract 2010, 16(3):574-579.

34. Anderson LA, Dedrick RF: Development of the Trust in Physician scale: a measure to assess interpersonal trust in patient-physician relationships. Psychol Rep 1990, 67(3 Pt 2):1091-1100.

35. Keller S: Zur Validität des Transtheoretischen Modells - Eine Untersuchung zur Veränderung des Ernährungsverhaltens [The validity of the transtheoretical model - 
a study on change of dietany behaviour], PhD thesis. Philipps-University Marburg, Department of Psychology; 1998. [in German, no English abstract available] http://archiv.ub.uni-marburg.de/diss/21998/0303/html/ (last accessed July 29, 2013).

36. Naik AD, Kallen MA, Walder A, Street RLJ: Improving hypertension control in diabetes mellitus: the effects of collaborative and proactive health communication. Circulation 2008, 117(11):1361-1368.

37. Simon D, Kriston L, Loh A, Spies C, Scheibler F, Wills C, Harter M: Confirmatory factor analysis and recommendations for improvement of the Autonomy-Preference-Index (API). Health Expect 2010, 13(3):234-243.

38. Bieber C, Nicolai J, Hartmann M, Blumenstiel K, Ringel N, Schneider A, Harter M, Eich W, Loh A: Training physicians in shared decision-making - who can be reached and what is achieved? Patient Educ Couns 2009, 77(1):48-54.

39. Bieber C, Muller KG, Blumenstiel K, Hochlehnert A, Wilke S, Hartmann M, Eich W: A shared decision-making communication training programme for physicians treating fibromyalgia patients: effects of a randomized controlled trial. J Psychosom Res 2008, 64(1):13-20.

40. Emmons KM, Rollnick S: Motivational interviewing in health care settings. Opportunities and limitations. Am J Prev Med 2001, 20(1):68-74.

41. Sim G, Wain T, Khong E: Influencing behaviour change in general practice - Part 2 - motivational interviewing approaches. Aust Fam Physician 2009, 38(12):986-989.

42. Donner-Banzhoff N, Altiner A: arriba-hausarzt.de [Cardiovascular risk calculator]. [German website, no English version available] http://www.arriba-hausarzt.de (last accessed July 29,2013)

43. German College of General Practitioners and Family Physicians (DEGAM): Patienteninformationen zur Vorbeugung von Herzinfarkt und Schlaganfal [Information for patientes to prevent heart attack and ischaemic stroke. [German website, no English version available] [http://leitlinien.degam.de/ index.php?id=250] (last accessed July 29, 2013).

44. Littell R, Milliken G, Stroup W, Wolfinger R, Schabenberger O: Chapter 8: Random Coefficient Models. NC, USA: SAS Institute Inc. Cary; 2006:317-342.

45. Chenot JF: Cluster randomised trials: an important method in primary care research. Z Evid Fortbild Qual Gesundhwes 2009, 103(7):475-480 [in German, English abstract available].

46. Tinsel I, Buchholz A, Vach W, Siegel A, Durk T, Loh A, Buchholz A, Niebling W, Fischer KG: Implementation of shared decision making by physician training to optimise hypertension treatment. Study protocol of a cluster-RCT. BMC Cardiovasc Disord 2012, 12(1):73.

47. The European Agency for the Evaluation of Medicinal Products, Committee for Propriety Medicinal Products: Points to consider on adjustment for baseline covariates. London: CPMP/EWP/2863/99; 2003:1-10.

48. Scholl I, Kriston L, Dirmaier J, Harter M: Comparing the nine-item Shared Decision-Making Questionnaire to the OPTION Scale - an attempt to establish convergent validity. Health Expect 2012. [http://onlinelibrary.wiley. com/doi/10.1111/hex.12022/pdf] (last accessed September 05, 2013).

49. Kasper J, Legare F, Scheibler F, Geiger F: Shared decision-making and communication theory: grounding the tango. Z Evid Fortbild Qual Gesundhwes 2010, 104(8-9):636-641 [in German, English abstract available].

50. Glynn LG, Murphy AW, Smith SM, Schroeder K, Fahey T: Interventions used to improve control of blood pressure in patients with hypertension. Cochrane Database Syst Rev 2010, 3, CD00518.

51. Sanders AR, van Weeghel I, Vogelaar M, Verheul W, Pieters RH, de Wit NJ, Bensing JM: Effects of improved patient participation in primary care on health-related outcomes: a systematic review. Fam Pract 2013, 30(4):365-378.

52. Conen D, Tschudi P, Martina B: Twenty-four hour ambulatory blood pressure for the management of antihypertensive treatment: a randomized controlled trial. J Hum Hypertens 2009, 23(2):122-129.
53. Dolan E, Stanton AV, Thom S, Caulfield M, Atkins N, Mclnnes G, Collier D, Dicker P, O'Brien E: Ambulatory blood pressure monitoring predicts cardiovascular events in treated hypertensive patients - an Anglo-Scandinavian cardiac outcomes trial substudy. J Hypertens 2009, 27(4):876-885.

54. Kikuya M, Hansen TW, Thijs L, Bjorklund-Bodegard K, Kuznetsova T, Ohkubo T, Richart T, Torp-Pedersen C, Lind L, Ibsen H, Imai Y, Staessen JA: Diagnostic thresholds for ambulatory blood pressure monitoring based on 10-year cardiovascular risk. Circulation 2007, 115(16):2145-2152.

doi:10.1186/1471-2296-14-135

Cite this article as: Tinsel et al:: Shared decision-making in antihypertensive therapy: a cluster randomised controlled trial. BMC Family Practice 2013 14:135.

\section{Submit your next manuscript to BioMed Central and take full advantage of:}

- Convenient online submission

- Thorough peer review

- No space constraints or color figure charges

- Immediate publication on acceptance

- Inclusion in PubMed, CAS, Scopus and Google Scholar

- Research which is freely available for redistribution

Submit your manuscript at www.biomedcentral.com/submit
C Biomed Central 